\title{
Alu-PCR: Characterization of a Chromosome 6-Specific Hybrid Mapping Panel and Cloning of Chromosome-Specific Markers
}

\author{
Eckart U. Meese, ${ }^{*}$ Paul S. Meltzer, † Paul W. Ferguson, " and Jeffrey M. Trent* $†$ \\ *University of Michigan Medical Center Departments of Radiation Oncology, †Pediatrics, and $¥$ Human Genetics, \\ MSRBII C560, 1150 West Medical Center Drive, Ann Arbor, Michigan 48109-0668
}

Received July 1, 1991; September 26, 1991

\begin{abstract}
The Alu-polymerase chain reaction (Alu-PCR) was applied to selectively amplify DNA sequences from human chromosome 6 using a single primer (A1) directed to the human Alu consensus sequence. A specific amplification pattern was demonstrated for a panel of eight somatic cell hybrids containing different portions of chromosome 6. This PCR pattern permits the identification of submicroscopic DNA alterations and can be utilized as a reference for additional chromosome 6-specific hybrids. To obtain new chromosome 6-specific markers we established two libraries from PCR-amplified sequences using two somatic cell hybrids (MCH381.2D and 640-5A). Out of a total of 109 clones that were found to be chromosome 6 specific, 13 clones were regionally assigned. We also included a procedure that allows the isolation of chromosome 6-specific markers from hybrids that contain human chromosomes other than 6 . Our results will contribute to the molecular characterization of chromosome 6 by fostering characterization of somatic cell hybrids and by the generation of new regionally assigned DNA markers. (c) 1992 Academic Press, Ine.
\end{abstract}

\section{INTRODUCTION}

The molecular analysis of human chromosomes requires a large number of chromosome-specific DNA probes. The saturation of chromosome regions with specific DNA markers has been an essential part of the identification of numerous disease-associated genes (Wallace et al., 1990; Riordan et al., 1989). DNA sequence amplification via the polymerase chain reaction (PCR) offers a novel and highly efficient approach to isolate single-copy DNA sequences from somatic cell hybrids containing specific human chromosomes (Nelson et al., 1989). A variety of PCR primers homologous to human repetitive sequences have been described to selectively amplify human DNA in human/rodent hybrids (Ledbetter et al., 1990b; Cotter et al., 1990). Alu sequences are the most abundant repetitive sequence in the human genome and have been successfully utilized to isolate human DNA sequences (Kariya et al., 1987). The use of PCR to identify DNA markers avoids both the construction and the screening of chromosome-specific DNA libraries from somatic cell hybrids.
The studies utilizing $A l u-P C R$ can be grouped into two categories. One approach uses Alu primers, which are directed to a site-specific Alu sequence (Epstein et al., 1990; Economou et al., 1990; Zuliani and Hobbs, 1990). In this approach, the amplification of site-specific sequences is achieved by the use of two specific primers or by a combination of one locus-specific primer and a second primer that hybridizes nonspecifically to human $A l u$ consensus sequences. Although this method has been demonstrated to be useful for the identification of polymorphisms, it requires prior knowledge of sequences flanking a specific Alu repeat. The second approach utilizes a nonspecific Alu primer directed to Alu consensus sequences (Ledbetter et al., 1990b). A single primer sequence allows the amplification of DNA sequences between two Alu repeats, which are in an inverted orientation and within a distance to be amplified by PCR (Brooks-Wilson et al., 1990). The major advantage of the second approach is that no prior sequence information about the amplified DNA and its flanking regions is needed. Furthermore, in contrast to human clones isolated from chromosome-specific libraries, the majority of sequences amplified by $A l u$-PCR are free of internal Alu repeats and can be readily used as a probe on Southern blots.

The primary application of Alu-PCR-amplified sequences is twofold. First, the amplified DNA fragments can be cloned and used as probes in Southern blots (Brooks-Wilson et al., 1990). The exact chromosomal location of these probes can be determined within a few hours by hybridization to Alu-PCR-amplified material derived from a somatic hybrid panel. Second, Alu-PCR can be used to characterize human rodent cell hybrids. Most recently, Ledbetter et al. (1990a), reported a chromosome-specific pattern of amplification products for a panel of somatic cell hybrids. Karyotyping by PCR is faster than conventional cytogenetic analysis and more sensitive for the identification of smaller chromosome pieces that cannot be detected by conventional cytogenetic methods.

In this study, we report the amplification of chromosome 6-specific sequences using two primers (A1 and A1S) recently described by Brooks-Wilson et al. (1990). 
The amplified sequences give rise to a fingerprint pattern that is specific for hybrids containing different portions of chromosome 6 . Amplified sequences were cloned from two hybrids with different parts of chromosome 6 , and DNA markers generated by this approach will facilitate mapping and characterization of chromosome 6.

\section{MATERIAL AND MËTHODS}

Hybrid cell lines. A detailed description of the hybrid panel is given elsewhere (Meese et al., 1992). In brief, the somatic cell hybrids used in this study are as follows. Hybrids Q998-1B and MCH262-A1D6 contain an entire chromosome 6 as the only human material in a rodent background. Hybrids MCH381.2D and $\mathrm{MCH} 381.1$ are radiation-reduced derivatives of MCH262-A1D6 containing $\operatorname{del}(6)(q 16 q 23)$ as their only human DNA. Hybrid 640-5A contains 6cen-qter, hybrid Q836-3A contains 6q13-qter, hybrid 5184-4 contains 6q21-qter, HAL26-12 contains 6pter-q13, and hybrid R21-1B contains $\operatorname{del}(6)(q 21 q 25)$ (see Meese et al., 1992).

Primers and PCR conditions. The PCR reaction $(100 \mu \mathrm{l})$ was carried out using primers $\mathrm{A} 1$ and A1S as described previously by Brooks-Wilson et al. (1990). Briefly, the primer concentration was 0.5 $\mu M$ in $50 \mathrm{mM}$ Tris-HCl pH $8.0,0.05 \%$ Tween $20,0.05 \% \mathrm{NP}-40$, and $1.3 \mathrm{mM} \mathrm{MgCl}_{2}$ for the A1 primer or $1.8 \mathrm{mM} \mathrm{MgCl}$ for the A1S primer, dNTPs at $200 \mu M$ each, and Thermus aquaticus polymerase $2.5 \mathrm{u}$ (Perklin-Elmer/Cetus). Somatic cell hybrid DNA was used at a concentration of $250 \mathrm{ng} /$ reaction, total rodent DNA at $250 \mathrm{ng} /$ reaction, and total human DNA at $20 \mathrm{ng} /$ reaction. Initial denaturation was $94^{\circ} \mathrm{C}$ for $3 \mathrm{~min}$ followed by 25 cycles of $94^{\circ} \mathrm{C}$ denaturation $(45 \mathrm{~s}), 58^{\circ} \mathrm{C}$ annealing ( $2 \mathrm{~min}$ ), and $72^{\circ} \mathrm{C}$ extension ( $45 \mathrm{~s}$ plus $10 \mathrm{~s} / \mathrm{cycle}$ increase) with a final extension of 10 min at $72^{\circ} \mathrm{C}$ in an aulomated thermal cycler (Perklin-Elmer/Cetus).

Gel electrophoresis and Southern blot nnalysis. The PCR-amplified sequences ( $25 \%$ of the $100 \mu$ l reaction) were size fractionated on a $2 \%$ agarose gel in Tris-borate-EDTA buffer using an automated gel system (Probe Tech 1 Oncor). Gels are run at $70 \mathrm{~V}$ for $3-5 \mathrm{~h}$, or overnight at $25 \mathrm{~V}$. Following photography, the DNA was denatured in $1 \mathrm{~N}$ $\mathrm{NaOH}$ for 30 min and transferred onto a Nylon membrane using vacuum blotting on the Probe Tech1 system. Random primer labeling (Feinberg and Vogelstein, 1984) and hybridization was performed as previously described (Meese et al., 1989). For the Southern blot analysis of genomic DNAs, 5-10 $\mu \mathrm{g}$ of DNA from each hybrid and $10 \mu \mathrm{g}$ of human and mouse DNA were digested for $12 \mathrm{~h}$ with $5 \mathrm{U} / \mu \mathrm{g}$ of restriction enzyme according to the manufacturer's instruction.

Cloning of Alu-PCR amplified sequences. To clone PCR products, DNA was amplified using the primer A1S, which is identical to A1 but contains a SalI site at its 5' end. DNA was amplified from two different hybrids: MCH381.2D, which contains del(6)(q16q23) and 640-5A, which contains 6cen-qter. Unincorporated nucleotides and primers were removed by a centrifugation through a centricon 30 filter for 15 min. The amplified sequences were phosphorylated and ligated to form concatamers as described recently (Jung et al., 1990). The formation of concatamers increases the efficiency of the subsequent SalI digest and permits monitoring of the digestion. Sall-digested PCR product ( $225 \mathrm{ng}$ ) was ligated to $75 \mathrm{ng}$ of SalI-digested and dephosphorylated pBluescript. DH5a competent cells were transformed with the ligation product and selected on NZY plates containing ampicillin (50 $\mu \mathrm{g} / \mathrm{ml})$ and X-Gal/IPTG).

\section{RESULTS}

\section{Alu-Based PCR Fingerprinting on Chromosome 6}

The primers Al and A1S were previously shown to selectively amplify human-specific sequences on chromosome 10 (Brooks-Wilson et al., 1990). As demonstrated in Fig. 1a, no amplification was found in total hamster or total mouse DNA. Amplification of chromo- some 6-specific hybrid DNAs yielded a variable number of PCR products that appeared as discrete bands on an ethidium bromide gel. However, amplification of total human DNA results in an overall smear on an ethidium bromide gel with few distinct bands (indicating a large number of PCR products). These results are consistent with previous studies and demonstrate the specific amplification of human DNA against a rodent background using the primer sequences A1 and A1S.

A discrete amplification pattern was observed for each hybrid containing a different portion of chromosome 6 (Fig. 1b). The specificity of the amplification pattern was confirmed in several independent experiments. The number of PCR products for each cell hybrid largely depended on the presence of human chromosomes in addition to chromosome 6 . The presence of several different human chromosomes accounts for the large number of PCR products found in the somatic cell hybrids $640-5 \mathrm{~A}$ and 5184-4. In general, considerably fewer amplified DNA fragments were observed in hybrids that contain chromosome 6 (or portions of chromosome 6) as their only human components (e.g., MCH262-A1D6, MCH381.2D, and MCH381.1).

The presence of human chromosome 6 as the only human component facilitated the interpretation of the PCR pattern. The most distinct banding pattern was found in two radiation reduction hybrids. The hybrids MCH381.1 and MCH381.2D contain very similar portions of chromosome $6(\operatorname{del}(6)(\mathrm{q} 16 \mathrm{q} 23))$ and exhibit an almost identical PCR banding pattern. These distinct patterns facilitate the identification of possible submicroscopic DNA rearrangements during extended cell culture.

\section{Cloning of PCR-Amplified Sequences}

To obtain chromosome-specific markers, DNA from the hybrids $640-5 \mathrm{~A}$ and MCH381.2D was amplified using the primer A1S, which contains a SalI restriction site. Prior to cloning, the PCR products were self-ligated to form long concatamers that could be easily digested by SalI.

To obtain DNA marker for the long arm of chromosome 6, we established an Alu-PCR library from hybrid 640-5A that contains $6 \mathrm{cen}$-qter. Approximately 2000 recombinant clones were generated. Since this hybrid also contains human chromosomes other than $6(9,10$, and Y) a modified screening procedure was applied for the identification of chromosome 6-specific markers. DNA from the hybrid MCH262-AID6, which contains an entire chromosome 6 as its only human component, was amplified by Alu-PCR. The amplified PCR product was labeled and hybridized against clones derived from the hybrid 640-5A. Out of 99 selected clones (with sizes ranging from $700 \mathrm{bp}$ to $2000 \mathrm{bp}$ ) 23 gave a positive hybridization signal on Southern blots (Fig. 2).

To verify the chromosome 6-specific origin of these clones, they were used as probes against a Southern blot that contained DNA from the hybrid mapping panel. To 

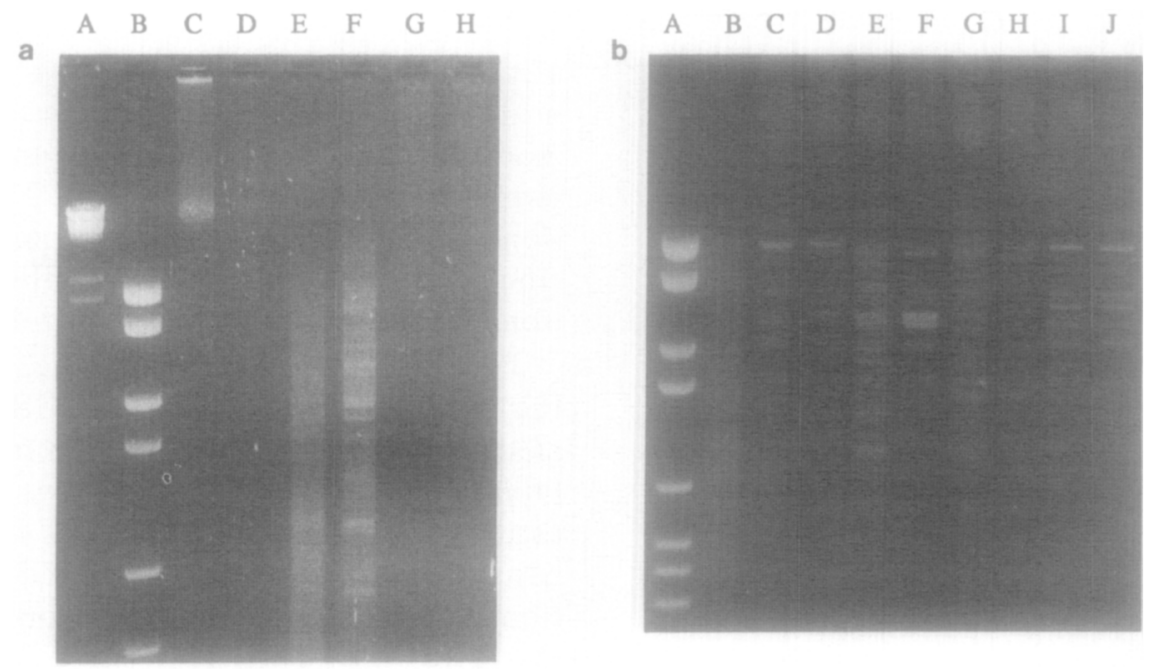

FIG. 1. (a) Gel electrophoresis of genomic DNA samples and Alu-PCR products. Lane A, HindIII-digested $\lambda$ DNA; Lane B, pBR328 digested by $B g l \mathrm{I}$ and $\mathrm{Hinfl}$; Lane C, nonrestricted total lymphocyte DNA (100 ng); Lane D, nonrestricted total lymphocyte DNA (10 ng); Lane E, total lymphocyte DNA amplified by Alu-PCR; Lane F, hybrid DNA (640-5A) amplified by Alu-PCR; Lane G, mouse DNA amplified by Alu-PCR; Lane H, hamster DNA (CHO) amplified by Alu-PCR. (b) Gel electrophoresis of Alu-PCR products. Lane A, pBR328 digested by Bgl and HinfI; Lane B, total human DNA; Lane C, hybrid Q998-1B; Lane D, hybrid MCH262-A1D6; Lane E, hybrid 640-5A; Lane F, hybrid Q836-3A; Lane G, hybrid 5184-4; Lane H, hybrid HAL-26; Lane I, hybrid MCH381.1; Lane J, hybrid MCH 381.2D.

enhance the signal intensity of the hybridized probe, DNA from the hybrid panel was amplified by Alu-PCR prior to gel electrophoresis and Southern transfer. As demonstrated in Fig. 3, strong hybridization signals were found within $3 \mathrm{~h}$ of exposure time. The hybridization pattern found with PCR-amplified genomic DNA can be grouped into two classes. The majority of the clones revealed multiple hybridization signals that were largely identical for different probes. As demonstrated in Fig. 3a, this hybridization pattern complicates the identification of the specific hybridization signal that indicates the location of the probe on chromosome 6 . The second group of probes identifies two major hybridization signals on Southern blots (Fig. 3b). These less

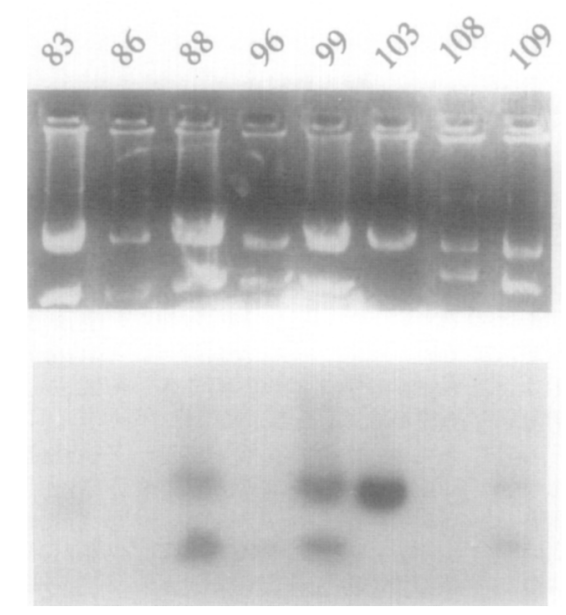

FIG. 2. Identification of chromosome 6-specific clones derived from somatic cell hybrid 640-5A (6cen-qter) (see text). Cloned PCR products were separated on an ethidium bromide gel (top), transferred, and hybridized with DNA from hybrid MCH262-A1D6, which contains an entire chromosome 6 as its only human chromosome (bottom). Prior to hybridization the chromosome 6 component of the hybrid MCH262-A1D6 was amplified by Alu-PCR. complex hybridization signals can readily be used to map these probes relative to our somatic hybrid panel. Using this approach two clones were regionally assigned on chromosome 6 (Fig. 5). In addition, five clones were mapped without prior amplification of the hybrid DNA (Table 1).

As an alternative source for clones, we established a second library from hybrid MCH381.2D. Approximately 3000 recombinant clones were generated. Since this hybrid contains chromosome 6 as its only human component clones were directly hybridized to a hybrid panel. Out of 10 clones that were confirmed to be chromosome 6 specific, 7 clones were regionally assigned on chromosome 6 (Figs. 4a, 4b, and 5). We hybridized a subset of clones against hybrid DNA without prior amplification of the hybrid DNA (Figs. 4a and 4b). Importantly, the regional assignment based on these experiments was found to be consistent with the results obtained with preamplified genomic DNA (Table 1).

\section{DISCUSSION}

In this study, we report the characterization of a chromosome 6-specific hybrid mapping panel by Alu-PCR. The study includes the analysis of monochromosomal hybrids and hybrids that contain other human chromosomes in addition to chromosome 6. Most recently, Ledbetter et al. (1990a) reported PCR-karyotyping for 16 monochromosomal hybrids containing different human chromosomes. Even though they reported minor variations between homologous chromosomes, the overall amplification pattern was largely consistent (Ledbetter et al., 1990a). Our analysis of chromosome 6 by Alu-PCR shows an almost identical amplification pattern for the radiation reduction hybrids that contain similar portions of chromosome 6 . The distinct pattern found in 


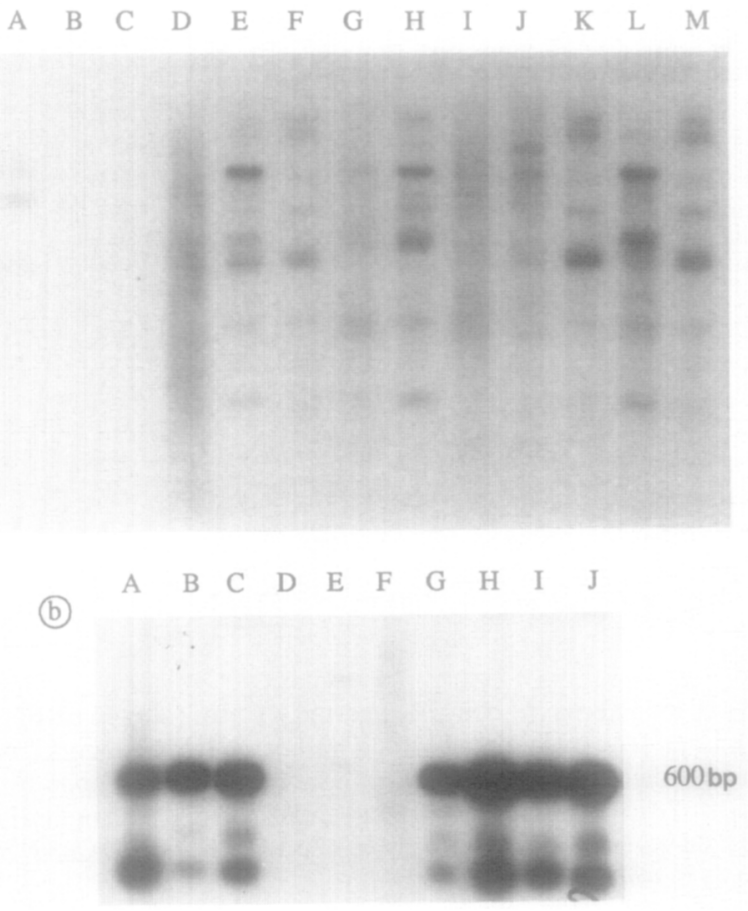

FIG. 3. Autoradiograph of a Southern blot that contains preamplified hybrid DNA. (a) Hybrid DNA was amplified by Alu-PCR, transferred, and hybridized against clone p108. The clone identifies multiple signals that are not probe specific. Lane A, HindIII digested $\lambda$ DNA; Lane B, hamster DNA; Lane C, mouse DNA; Lane D, total human DNA; Lane E, Q988-1B; Lane F, MCH262-A1D6; Lane G, 640-5A; Lane H, Q836-3A; Lane I, 5184-4; I ane J, HAL26-12; Lane K, MCH381.1; Lane L, R21-1B; Lane M, MCH381.2D. (b) Preamplified hybrid DNA was hybridized against clone H9.14, which identifies a probe-specific hybridization pattern. Lane A, total human DNA; Lane B, Q988-1B; Lane C, MCH262-A1D6; Lane D, 640-5A; Lane E, Q8363A; Lane F, 5184-4; Lane G, HAL26-12; Lane H, MCH381.1; Lane I. R21-1B; Lane J, MCH381.2D.

radiation reduction hybrids may facilitate monitoring possible submicroscopic DNA rearrangements using Alu-PCR.

As expected, the presence of additional human chromosomes was found to increase the number of PCR products and complicate the interpretation of the PCR banding pattern. However, the selected $\mathrm{PCR}$ primers give rise to an individual amplification pattern, which can be used to identify any particular hybrid within the panel. The hybrid-specific PCR pattern permits the identification of chromosomal changes that are known to occur frequently in somatic cell hybrids particularly after several cycles of freezing and thawing. Monitoring chromosomal changes by PCR is significantly faster than conventional cytogenetic analysis. Furthermore, Alu-PCR permits the identification of smaller chromosomal rearrangements that cannot be identified by cytogenetic means.

Our results also indicate certain limitations of AluPCR for the analysis of human rodent hybrid cells. As demonstrated in Fig. $1 \mathrm{~b}$, there are significantly more bands in the radiation reduction hybrids MCH381.1 and MCH381.2D than in the parental MCH262A1 hybrid that contains an entire chromosome 6 . These findings can be explained by a more efficient PCR amplification of the remaining $\mathrm{PCR}$ templates in the reduction hybrids. As the number of possible templates is reduced, more efficient amplification of any given template is found (Ledbetter et al., 1990a). Despite these limitations, PCR-based fingerprinting of chromosome 6-specific hybrids will substantially facilitate the characterization of additional hybrids containing chromosome 6 .

There are several new approaches that will increase the potential applications of PCR-based chromosome analysis. First, a variety of different primers directed to human repetitive sequences allows the efficient amplification of human DNA sequences of varying sizes (Ledbetter et al., 1990b). Second, the development of radiation reduction hybrids, which contain only very small fragments of a particular chromosome, facilitates the detailed analysis of selected chromosomal region by PCR (Benham et al., 1989). Third, digestion of DNA fragments prior to the amplification by PCR significantly reduces the complexity of the PCR banding pattern (Guzzeta et al., 1991). These new developments permit detailed PCR-based fingerprinting of any given chromosome region. Based on the amplification pattern, chromosomal rearrangements can be identified without cytogenetic analysis or conventional Southern blot analysis.

As demonstrated in this study, the amplified sequences can readily be cloned and used as chromosome region-specific markers. There are several advantages of Alu-PCR libraries over conventional chromosome-specific libraries. Depending on the source of DNA (e.g., radiation reduction hybrids) $A l u$-PCR can be applied to isolate clones from a very specific region. Furthermore, human chromosome-specific Alu-PCR libraries could be established from flow-sorted chromosomes eliminating the need for large amounts of starting material (Cotter $e t$ al., 1989).

TABLE 1

\begin{tabular}{|c|c|c|c|c|}
\hline Probe & $\operatorname{Size}^{\alpha}$ & Location & Origin $^{b}$ & Mapping method ${ }^{c}$ \\
\hline H9.4 & 1030 & $6 p$ & $\mathrm{MCH}$ & Amplified \\
\hline H9.5 & 1950 & $6 \mathrm{p}$ & $\mathrm{MCH}$ & Amplified/genomic \\
\hline H9.14 & 550 & $6 \mathrm{p}$ & $\mathrm{MCH}$ & Amplified \\
\hline $\mathrm{p} 88$ & 1125 & $6 q 14-q 16$ & $640-5 \mathrm{~A}$ & Genomic \\
\hline H9.7B & 1700 & 6q14-q16 & $\mathrm{MCH}$ & Amplified/genomic \\
\hline H9.12 & 770 & $6 q 14-q 16$ & MCH & Amplified \\
\hline H9.1 & 1400 & $6 q 14-q 16$ & $\mathrm{MCH}$ & Amplified \\
\hline H9.17 & 980 & $6 q 25.3-q 27$ & $\mathrm{MCH}$ & Amplified \\
\hline p122 & 790 & 6q25.3-q27 & $640-5 A$ & Amplified \\
\hline p99 & 1050 & $6 \mathrm{q} 24-\mathrm{q} 25.2$ & $640-5 \mathrm{~A}$ & Amplified/genomic \\
\hline p111 & 1200 & $6 q 24-q 25.2$ & $640-5 A$ & Genomic \\
\hline p68 & 1225 & $6 \mathrm{q} 24-\mathrm{q} 25.2$ & $640-5 \mathrm{~A}$ & Genomic \\
\hline p68u & 790 & $6 q 21-q 23$ & $640-5 \mathrm{~A}$ & Genomic \\
\hline
\end{tabular}

a The probe sizes are given in basepairs.

${ }^{b}$ The clones were isolated from two different libraries, which were derived from hybrid 640-5A and hybrid MCH381.2D, respectively.

'Clones were mapped using genomic DNA and amplified DNA. 

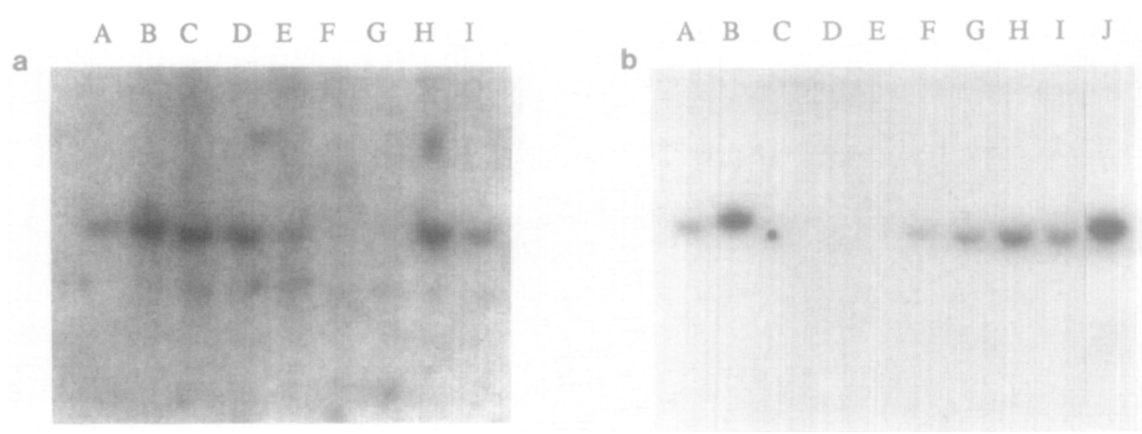

FIG. 4. Autoradiograph of a Southern blot containing nonamplified hybrid DNA. (a) The hybrid DNA was probed with clone H.1.11, which was mapped to 6q25.3-q27. Lane A, Q988-1B; Lane B, MCH262-A1D6; Lane C, 640-5A; Lane D, Q836-3A; Lane E, 5184-4; Lane F, HAL26-12; Lane G, MCH381.1; Lane H, R21-1B; Lane I, MCH381.2D. (b) The blot was hybridized with clone H9.5, which was mapped to 6p. Lane A, Q988-1B; Lane B, MCH262-A1D6; Lane C, 640-5A; Lane D, Q836-3A; Lane E, 5184-4; Lane F, HAL26-12; Lane G, MCH381.1; Lane H, R21-1B; Lane I, MCH381.2D; Lane J, total human DNA.

To summarize, an Alu-based fingerprint for chromosome 6 has been obtained and it demonstrates a chromosome-specific amplification pattern that will greatly facilitate further analysis of this chromosome. In addition, amplified sequences have been selectively cloned from

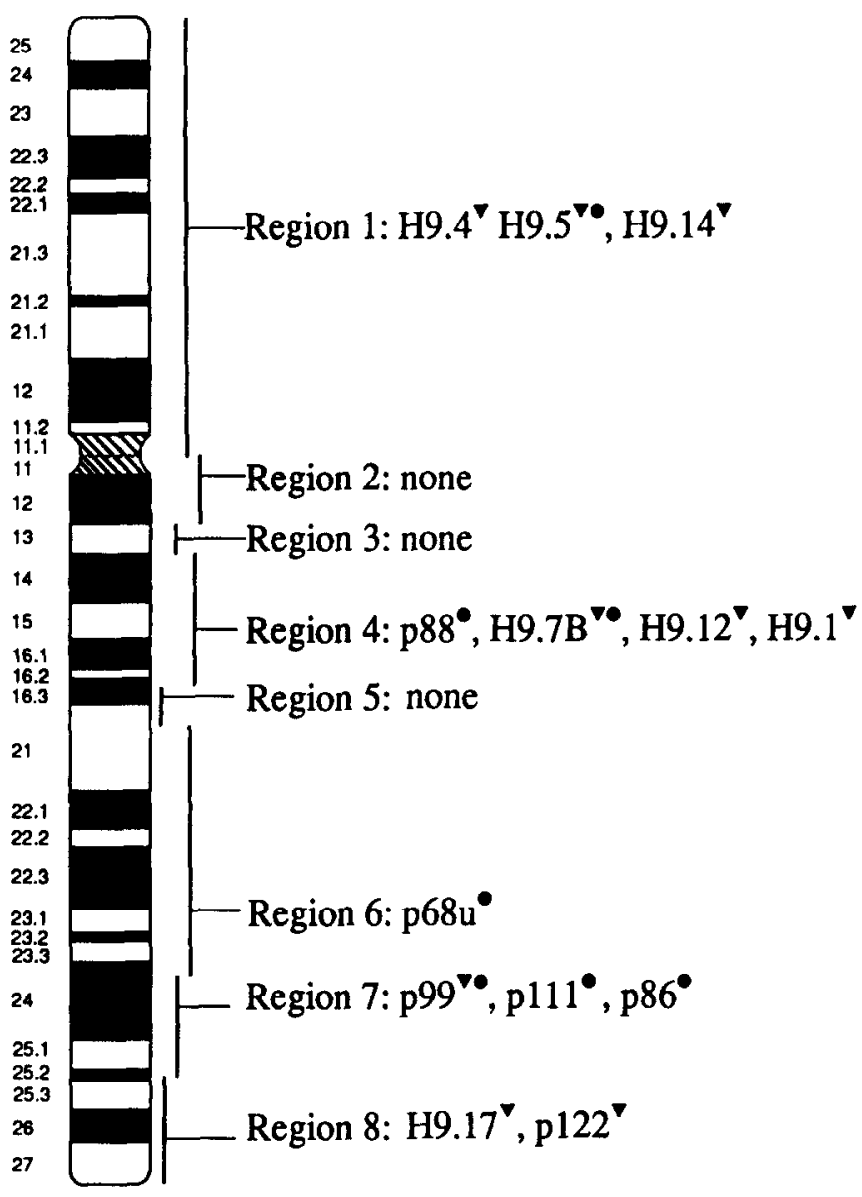

6

FIG. 5. Ideogram of chromosome 6 and regional distribution of cloned Alu-PCR products. This previously described hybrid mapping panel (Meese et al., 1991) divides chromosome 6 into 8 distinct regions, which are indicated by solid lines. The triangles identify probes wrapped by hybridization to amplified DNA; the circles identify probes mapped by hybridization to genomic DNA. human/rodent hybrids and utilized as region-specific DNA markers.

\section{ACKNOWLEDGMENTS}

We acknowledge the excellent technical assistance of Mr. Arthur Glatfelter. The hybrids R21-1B, 640-5A, Q998-1B, and 836-3A were a generous gift from Dr. Carol Jones (Eleanor Roosevelt Institute, Denver, CO). The hybrids MCII262-A1D6, MCH381.2D, and MCH381.1 were obtained from Dr. Eric Stanbridge (University of California-Irvine, CA). This research was supported in part by PHHS Grant CA-29476 (J.M.T.), awarded by the National Cancer Institute, Bethesda, MD.

\section{REFERENCES}

Benham, F., Hart, K., Crolla, J., Bobrow, M., Francavilla, M., and Goodfellow, P. N. (1989). A method for generating hybrids containing nonselected fragments of human chromosomes. Genomics 4: 509-517.

Brooks-Wilson, A. R., Goodfellow, P. N., Povey, S., Nevanlinna, H. A., de Jong, P. J., and Goodfellow, P. J. (1990). Rapid cloning and characterization of new chromosome 10 DNA markers by Alu element-mediated PCR. Genomics 7: 614-620.

Cotter, F. E., Hampton, G. M., Nasipuri, S., Bodmer, W. F., and Young, B. D. (1990). Rapid isolation of human chromosome-specific DNA probes from a somatic cell hybrid. Genomics 7: 257-263.

Cotter, F., Nasipuri, S., Lam, G., and Young, B. D. (1989). Gene mapping by enzymatic amplification from flow-sorted chromosomes. Genomics 5: 470-474.

Economou, E. P., Bergen, A. W., Warren, A. C., and Antonarakis, S. E. (1990). The polydeoxyadenylate tract of Alu repetitive elements is polymorphic in the human genome. Proc. Natl. Acad. Sci. USA 87: 2951-2954.

Epstein, N., Nahor, O., and Silver, J. (1990). The $3^{\prime}$ ends of Alu repeats are highly polymorphic. Nucleic Acids Res. 18: 4634.

Feinberg, A., and Vogelstein, B. (1984). Addendum: A technique for Radiolabeling DNA restriction fragments to a high specific activity. Anal. Biochem. 137: 66-67.

Guzzeta, V., Montes de Oca-Luna, R., Lupski, J. R., and Patel, P. I. (1991). Isolation of region-specific and polymorphic markers from chromosome 17 by restricted Alu polymerase chain reaction. Genomics 9: $31-36$.

Jung, V., Pestka, S. B., and Pestka, S. (1990). Efficient cloning of PCR generated DNA containing terminal restriction endonuclease recognition sites. Nucleic Acids Res. 18: 1656.

Kariya, Y., Kato, K., Hayashizaki, Y., Himeno, S., Tarui, S., and Kenichi, M. (1987). Revision of consensus sequence of human Alu repeats-A review. Gene 53: 1-10. 
Ledbetter, S. A., Garcia-Heras, J., and Ledbetter, D. H. (1990a). "PCR-karyotype" of human chromosomes in somatic cell hybrids. Genomics 8: 614-622.

Ledbetter, S. A., Nelson, D. L., Warren, S. T., and Ledbetter, D. H. (1990b). Rapid isolation of DNA probes within specific chromosome regions by interspersed repetitive sequence polymerase chain reaction. Genomics 6: $475-481$.

Meese, E. U., Meltzer, P. S., Witkowski, C. M., and Trent, J. M. (1989). Molecular mapping of the oncogene MYB and rearrangements in malignant melanoma. Genes Chrom. Cancer 1: 88-94.

Meese, E. U., Zogghbi, H. Y., Witkowski, C. M., Stanbridge, F. J., and Trent, J. M. (1992). Development and utilization of a somatic cell hybrid mapping panel to assign NotI linking probes to the long arm of human chromosome 6. Genomics 12: 542-548.
Nelson, D. L., Ledbetter, S. A., Corbo, L., Victoria, M. F., Ramirez-Solis, R., Webster, T. D., Ledbetter, D. H., and Caskey, C. T. (1989). Alu polymerase chain reaction: A method for rapid isolation of human-specific sequences from complex DNA sources. Proc. Natl. Acad. Sci. 86: 6686-6690.

Riordan, J. R., et al. (1989). Identification of the cystic fibrosis gene: Cloning and characterization of complementary DNA. Science 245: 1066-1073.

Wallace, M. R., Marchuk, D. A., Andersen, L. B., Letcher, R., et al. (1990). Type 1 neurofibromatosis gene: Identification of a large transcript disrupted in three NF1 patients. Science 249: 181-186.

Zuliani, G., and Hobbs, H. (1990). A high frequency of length polymorphisms in repeated sequences adjacent to Alu sequences. Am.J. Hum. Genet. 46: 1963-969. 